\title{
Antigenic Properties of the Human Immunodeficiency Virus Envelope during Cell-Cell Fusion
}

\author{
CATHERINE M. FINNEGAN, ${ }^{1,2}$ WERNER BERG, ${ }^{1,2}$ GEORGE K. LEWIS, ${ }^{1}$ AND ANTHONY L. DEVICO ${ }^{1 *}$ \\ Institute of Human Virology, University of Maryland Biotechnology Institute, ${ }^{1}$ and Department of Microbiology and \\ Immunology, University of Maryland School of Medicine, ${ }^{2}$ Baltimore, Maryland 21201
}

Received 1 February 2001/Accepted 27 July 2001

\begin{abstract}
Human immunodeficiency virus (HIV) fusion and entry involves sequential interactions between the viral envelope protein, gp120, cell surface CD4, and a G-protein-coupled coreceptor. Each interaction creates an intermediate gp120 structure predicted to display distinct antigenic features, including key functional domains for viral entry. In this study, we examined the disposition of these features during the fusion of HeLa cells expressing either $\mathrm{HIV}_{\mathrm{HXB} 2}$ envelope (Env cells) or CXCR4 and CD4 (target cells). Cell-cell fusion, indicated by cytoplasmic dye transfer, was allowed to progress for various times and then arrested. The cells were then examined for reactivity with antibodies directed against receptor-induced epitopes on gp120. Analyses of cells arrested by cooling to $4^{\circ} \mathrm{C}$ revealed that antibodies against the CD4-induced coreceptor-binding domain, i.e., 17b, 48d, and CG10, faintly react with Env cells even in the absence of target cell or soluble CD4 (sCD4) interactions. Such reactivity increased after exposure to $\mathrm{SCD} 4$ but remained unchanged during fusion with target cells and was not intensified at the Env-target cell interface. Notably, the antibodies did not react with Env cells when treated with a covalent cross-linker either alone or during fusion with target cells. Immunoreactivity could not be promoted or otherwise altered on either temperature arrested or cross-linked cells by preventing coreceptor interactions or by using a 17b Fab. In comparison, two other gp120-CD4 complexdependent antibodies against epitopes outside the coreceptor domain, 8F101 and A32, exhibited a different pattern of reactivity. These antibodies reacted with the Env-target cell interface only after 30 min of cocultivation, concurrent with the first visible transfer of cytoplasmic dye from Env to target cells. At later times, the staining surrounded entire syncytia. Such binding was entirely dependent on the formation of gp120-CD4CXCR4 tricomplexes since staining was absent with SDF-treated or coreceptor-negative target cells. Overall, these studies show that access to the CD4-induced coreceptor-binding domain on gp120 is largely blocked at the fusing cell interface and is unlikely to represent a target for neutralizing antibodies. However, new epitopes are presented on intermediate gp120 structures formed as a result of coreceptor interactions. Such findings have important implications for HIV vaccine approaches based on conformational alterations in envelope structures.
\end{abstract}

Human immunodeficiency virus (HIV) entry occurs through a $\mathrm{pH}$-independent mechanism involving the direct fusion of virus and cell membranes. The viral envelope proteins that mediate this process include a soluble glycoprotein component, gp120, and transmembrane subunit, gp41, which are associated by noncovalent interactions and assembled into trimeric spikes on the virion surface. In the currently accepted model of HIV infection, the entry process begins with the binding of gp120 to cell surface CD4. This interaction forms a gp120-CD4 complex that expresses a binding site for certain $\mathrm{CC}$ or $\mathrm{CxC}$ chemokine receptors on the gp120 component (33). The major chemokine receptor, or coreceptor, used by macrophage-tropic (or R5) HIV strains is CCR5 (1), whereas T-tropic (or X4) viruses predominantly use CXCR4 (8). Contact between coreceptor and the gp120-CD4 complex forms a tripartite intermediate that is thought to dislocate gp120 from gp41 (30). Consequently, gp41 undergoes a conformational change exposing an amino-terminal hydrophobic peptide that inserts into the target cell membrane. The gp41 trimers rapidly acquire a coiled-coil transitional conformation that mediates

* Corresponding author. Mailing address: Institute of Human Virology, 725 W. Lombard St., N649, Baltimore, MD 21201. Phone: (410) 706-4680. Fax: (410) 706-4694. E-mail: devico@umbi.umd.edu. fusion of viral and cell membranes and delivery of the virus core to the target cell cytoplasm $(2,4)$.

Because of their unique structures, HIV envelope intermediates have the potential to elicit distinct immune responses, possibly including broadly neutralizing antibodies. Recent evidence with either subunit or cell-based immunogens supports this concept $(5,17)$. One array of such epitopes is induced on gp120 by CD4 binding and is specific to the gp120-CD4 complex. Some of these epitopes comprise the coreceptor-binding domain and are being considered as potentially important targets for antibodies to inhibit virus-mediated membrane fusion. However, despite antibody recognition of these epitopes on soluble gp120-CD4 complexes, it is unclear whether such reactivity occurs in the context of cell-cell or virus-cell membrane fusion. Monoclonal antibodies (MAbs) against conserved CD4-induced epitopes potently block soluble CD4 (sCD4)activated fusion with target cells expressing coreceptor alone but have minimal effects in the standard cell fusion system using target cells expressing both CD4 and coreceptor (23). Other antibodies raised against gp120-CD4 complexes are either poorly neutralizing (5) or variably enhance or inhibit infection, depending on the assay conditions $(18,25)$. Therefore, the successful development of effective immunogens based on altered HIV envelope structures must consider the antigenic 
nature of gp120 intermediates as they appear during the progression of HIV-mediated fusion.

In order to address this question, we developed an assay system that simultaneously visualizes cell-cell fusion and MAb immunoreactivity with various domains on intermediate HIV envelope structures. In this study, we show that CD4-induced epitopes within the coreceptor-binding domain exhibit limited exposure on envelope-expressing cells even in the absence of CD4. However, these epitopes appear to be restricted from interactions with cognate MAbs at a fusing cell interface where envelope encounters CD4. In contrast, epitopes characterized here as specific for gp120-CD4-coreceptor tricomplexes are accessible to cognate MAbs at the cell-cell interface and on the surfaces of developing syncytia.

\section{MATERIALS AND METHODS}

Cells. The HeLa/CD4/MAGI and the U373/CD4/MAGI cell lines were provided by Michael Emerman through the AIDS Research and Reference Reagent Program, Division of AIDS, National Institute of Allergy and Infectious Diseases, National Institutes of Health. These cells contain an HIV long terminal repeat (LTR)-driven $\beta$-galactosidase gene that is activated by HIV tat expression (15). The HeLa cells endogenously express CXCR4 (8). The cell lines were maintained in Dulbecco modified Eagle medium (DMEM; Gibco-BRL) supplemented with $10 \%$ heat-inactivated fetal bovine serum (FBS), 2 mM L-glutamine, antibiotics, $0.1 \mathrm{mg}$ of G418 (Gibco-BRL)/ml, and $0.05 \mathrm{mg}$ of hygromycin $\mathrm{B} / \mathrm{ml}$ (complete medium). The stable HeLa cell line (Env cells) producing replicationdefective HXB2 virions was generated by cotransfection of HeLa cells with the genetic constructs HIV-gpt and HXB2-env. The HIV-gpt construct contains the HXB2 proviral genome with the gp160-coding region removed and substituted by the bacterial gpt gene driven by the simian immunodeficiency virus 40 promoter to allow selection by mycophenolic acid. These cells were maintained in DMEM containing $10 \%$ FBS, and $50 \mu \mathrm{g}$ of mycophenolic acid and $50 \mu \mathrm{g}$ of gentamicin/ml. To monitor consistent levels of HXB2 envelope expression, cells were labeled with the anti-gp120 MAb 2G12 and analyzed by flow cytometry.

Antibodies and reagents. The human MAbs 17b, A32, and 48d, derived from HIV type 1 (HIV-1)-infected individuals in the United States, were provided by James Robinson, Tulane University, New Orleans, La. MAb 8F101 was provided by Ranajiit Pal, Advanced BioScience Lab, Inc., and MAb CG10 was provided by Jonathan Gershoni, Tel Aviv University. MAb 2G12 was obtained from Hermann Katinger, IAM Pharmaceuticals, Inc., Vienna, Austria. The secondary antibodies goat anti-mouse and goat anti-human conjugated to Alexa 594, calcein-AM, and CellTracker Green CMFDA were obtained from Molecular Probes, Eugene, Oreg. Bis-sulfosuccinimidylsuberate $\left(\mathrm{BS}^{3}\right)$ was obtained from Pierce (Rockford, Ill.). sCD4 was a generous gift from Werner Meier at Biogen, Cambridge, Mass. The 17b Fab fragments were generated according to the manufacturer's protocols by using the ImmunoPure Fab Preparation Kit from Pierce. 17b Fab fragments were directly conjugated to Alexa 594 by using an Alexa 594 protein labeling kit obtained from Molecular Probes. T21 was synthesized according to the published sequence (32) by The Biopolymer Laboratory, University of Maryland, Baltimore.

Cell fusion assay. Env cells $\left(5 \times 10^{6}\right.$ to $\left.10 \times 10^{6}\right)$ were labeled by suspension in prewarmed $\left(37^{\circ} \mathrm{C}\right)$ serum-free DMEM, containing $0.33 \mu \mathrm{M}$ CellTracker Green CMFDA (absorption, $492 \mathrm{~nm}$; emission, $516 \mathrm{~nm}$ ), for $30 \mathrm{~min}$ at $37^{\circ} \mathrm{C}$ in a $5 \% \mathrm{CO}_{2}$ incubator. The cells were then pelleted by centrifugation and resuspended in fresh prewarmed complete medium for $30 \mathrm{~min}$ at $37^{\circ} \mathrm{C}$ in a $5 \% \mathrm{CO}_{2}$ incubator. The cells were washed three times with phosphate-buffered saline (PBS) and resuspended at a final concentration of $5 \times 10^{5}$ cells $/ \mathrm{ml}$ in complete medium. In the standard fusion system, HeLa/CD4/MAGI cells (target cells) were seeded at maximal density on glass coverslips overnight. To perform control experiments in the absence of coreceptor, U373/CD4/MAGI cells, which do not express CXCR4, were used instead. Labeled Env cells $\left(10^{5}\right.$ in $\left.200 \mu \mathrm{l}\right)$ were added to each coverslip and incubated for intervals of 0 to $120 \mathrm{~min}$, as indicated in the text, at $37^{\circ} \mathrm{C}$ in a $5 \% \mathrm{CO}_{2}$ incubator. Cell-cell fusion intermediates were arrested and fixed by adding $\mathrm{BS}^{3}$ to a final concentration of $1 \mathrm{mM}$. After $15 \mathrm{~min}$ at room temperature, the fixing process was stopped by the addition of $20 \mathrm{mM}$ Tris ( $\mathrm{pH}$ 7.4) for $15 \mathrm{~min}$ at room temperature. Alternatively, the fusion intermediates were arrested by chilling cells to $4^{\circ} \mathrm{C}(10,11,12,14,19)$ rather than by fixation. The coverslips were rinsed three times in $4^{\circ} \mathrm{C}$ PBS and placed on ice. Nonspecific antibody binding was blocked by incubating the coverslips in $2 \%$ normal goat serum (NGS) for $30 \mathrm{~min}$ on ice. The coverslips were incubated with $5 \mu \mathrm{g}$ of primary MAbs/ml, unless otherwise indicated, in PBS containing 2\% NGS for $1 \mathrm{~h}$ on ice. To fix both the primary antibody and the cytoplasmic CellTracker Green, coverslips were rinsed three times with $4^{\circ} \mathrm{C}$ PBS, fixed in ice-cold $4 \%$ paraformaldehyde (PFA) for $1 \mathrm{~min}$, and permeabilized in chilled methanol for $10 \mathrm{~min}$. After rehydration in PBS, the cells were incubated with goat anti-mouse or goat anti-human secondary antibodies coupled to Alexa 594 (absorption, $590 \mathrm{~nm}$; emission, $617 \mathrm{~nm}$ ) at $5 \mu \mathrm{g} / \mathrm{ml}$ for $30 \mathrm{~min}$ at room temperature. The emission spectrum of Alexa 594 does not interfere with the emission wavelength of CellTracker Green. After 3 rinses in PBS, the cells were mounted in Vectashield (Vector Labs, Burlingame, Calif.) for microscopic analysis. Images were taken on a Zeiss LSM410 confocal microscope at $\times 100$ magnification. Nomarski images and images obtained with each fluorescent dye were acquired separately and later superimposed by using Adobe Photoshop to provide a composite view of selected fields.

In experiments to estimate interference by shed envelope, $5 \times 10^{5}$ labeled Env cells were incubated in $1 \mathrm{ml}$ of complete medium at $37^{\circ} \mathrm{C}$ for $2 \mathrm{~h}$. The Env cells were then removed by centrifugation, and $200 \mu \mathrm{l}$ of the conditioned medium was applied to coverslips of plated $\mathrm{HeLa} / \mathrm{CD} 4 / \mathrm{MAGI}$ cells. The target cells were incubated for $2 \mathrm{~h}$ at $37^{\circ} \mathrm{C}$ in $0.1 \%$ sodium azide, fixed with $\mathrm{BS}^{3}$, and then stained with MAbs as described above.

sCD4 binding assay. For assays involving sCD4, Env cells were seeded on $22-\mathrm{mm}^{2}$ glass coverslips for $24 \mathrm{~h}$ at maximal density of $7.5 \times 10^{5}$ cells per coverslip. Next, $200 \mu \mathrm{l}$ of $1 \mu \mathrm{g}$ of sCD4/ml in DMEM was added per coverslip for $120 \mathrm{~min}$ at $4^{\circ} \mathrm{C}$. Cells were fixed with $\mathrm{BS}^{3}$ and stained as described in the cell-cell fusion assay. For some experiments, sCD4-triggered Env cells were rapidly cooled to $4^{\circ} \mathrm{C}$ without fixation and stained as described above.

Inhibition of coreceptor binding. CellTracker Green-labeled Env cells and $\mathrm{HeLa} / \mathrm{CD} 4 / \mathrm{MAGI}$ target cells were treated with $3 \mu \mathrm{g}$ of stromal-cell-derived factor $1 \beta(\mathrm{SDF}-1 \beta) / \mathrm{ml}$ for $1 \mathrm{~h}$ at $37^{\circ} \mathrm{C}$ and then cocultivated at $37^{\circ} \mathrm{C}$ for the indicated times as described above but in the presence of $3 \mu \mathrm{g}$ of chemokine $/ \mathrm{ml}$. Cell-cell fusion was arrested by fixation with $\mathrm{BS}^{3}$ or by rapid cooling to $4^{\circ} \mathrm{C}$. Immunostaining was performed as described above with MAbs at $5 \mu \mathrm{g} / \mathrm{ml}$, unless otherwise indicated. Images were taken on a Zeiss LSM410 confocal microscope. Nomarski images and images for each fluorescent dye were acquired separately and superimposed by using Adobe Photoshop.

Neutralization of cell-cell fusion. Target cells $\left(10^{4}\right)$ were added to wells in a 96-well microtiter plate (Falcon, Lincon Park, N.J.) and incubated overnight at $37^{\circ} \mathrm{C}$ in $200 \mu \mathrm{l}$ of complete medium. Env cells $\left(10^{4}\right)$ were treated with the indicated amount of antibody and then cocultured with target cells in the presence of antibody for $18 \mathrm{~h}$ at $37^{\circ} \mathrm{C}$. The cells were then washed in PBS, and syncytium formation was quantified as a function of tat-mediated $\beta$-galactosidase production in the target cells. $\beta$-Galactosidase was quantified as a function of activity by using a chemiluminescence reagent (Galactostar; Tropix, Bedford, Mass.), according to the manufacturer's protocol. The resulting chemiluminescence was quantified by using a Victor fluorescence plate reader (EG\&G Wallac, Gaithersburg, Md.).

\section{RESULTS}

Cell-cell fusion and syncytium formation mediated by the HIV envelope in a HeLa cell-based system. HIV-mediated cell-cell fusion has been successfully visualized and characterized by using diffusible intracellular dyes (29). We therefore incorporated this approach into a fusion assay system based on two types of HeLa cells, one expressing human CD4 (target cells) and the other expressing stable and consistent cell surface levels of the HIV HXB2 envelope (Env cells). Since this envelope uses the CXCR4 coreceptor endogenously expressed on HeLa cells, the system provides all of the surface components necessary for HIV-mediated fusion. Env cells were loaded with a fixable cytoplasmic dye, CellTracker Green, and cocultured with a monolayer of target cells. Ensuing cell-cell interactions were then allowed to progress for various lengths of time and arrested by either cooling the system to $4^{\circ} \mathrm{C}$ or by fixing cells with the homobifunctional cross-linker, $\mathrm{BS}^{3}$. The former method inhibits membrane mobility, the spread of cellcell contact, and the multiplication of gp120-CD4 interactions (10) and was recently used to successfully characterize the 


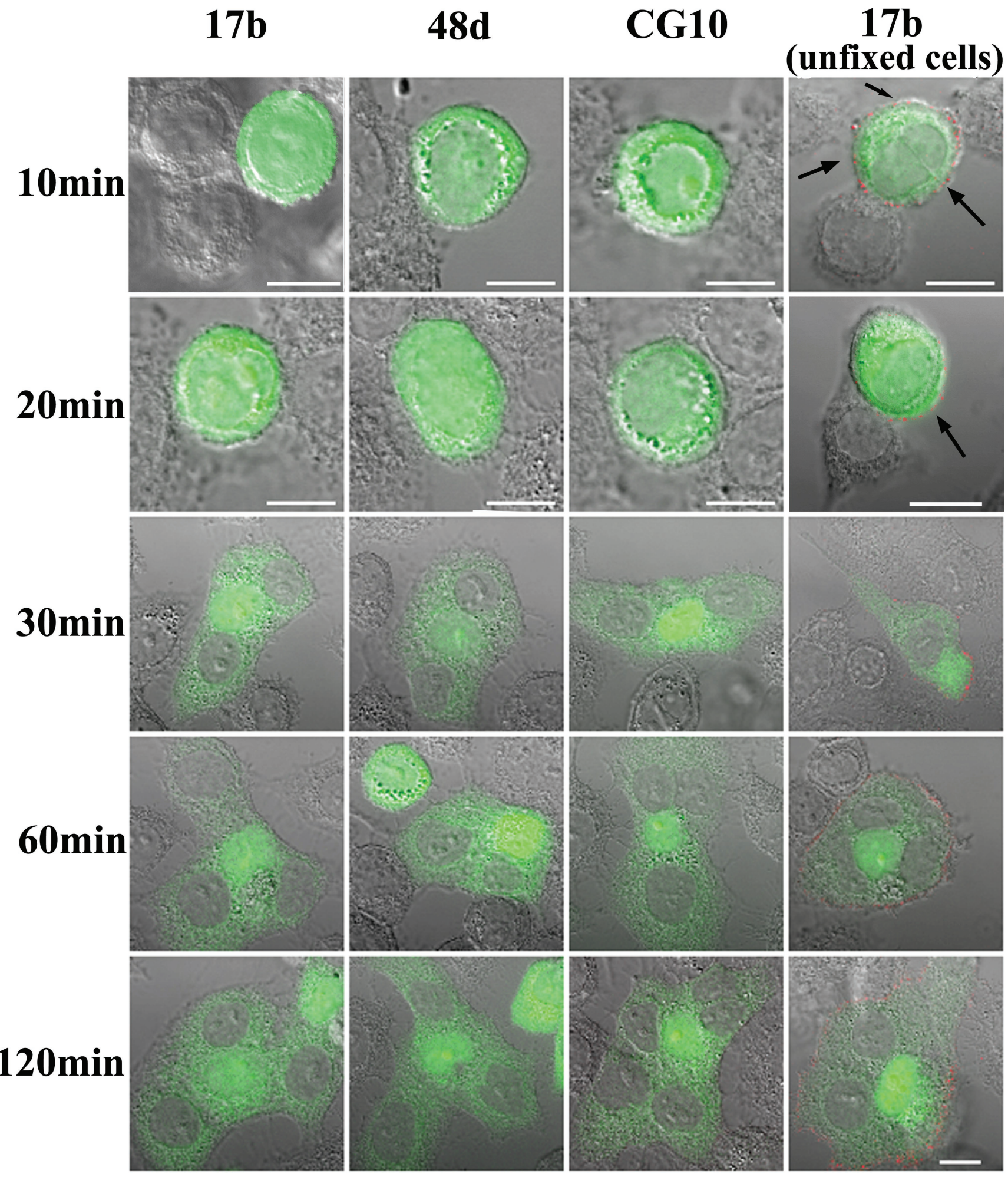

FIG. 1. MAb reactivity with epitopes in the gp120 coreceptor-binding domain during cell-cell fusion. CellTracker Green-labeled Env cells were cocultivated with target cells for the indicated times as described in Materials and Methods. Immunostaining was performed with BS ${ }^{3}$-fixed cells or with unfixed cells cooled to $4^{\circ} \mathrm{C}$ (noted at right) as described in Materials and Methods. The indicated MAbs were used at $5 \mu \mathrm{g} / \mathrm{ml}$. Cell nuclei appear as gray areas in stained syncytia. Fusion of Env and target cells produces the light green cytoplasmic staining surrounding the nuclei. All antibodies were tested in parallel along with human isotype controls, which produced no binding signal (data not shown). Reactivity was detected only with the unfixed cells (right); due to the faint signal, the images were digitally enhanced for clarity. Arrows indicate areas of Env cell staining that are not in contact with target cells. Representative images are shown. Each experiment was repeated at least three times with the same results. Scale bar, $10 \mu \mathrm{m}$. 
$17 b$

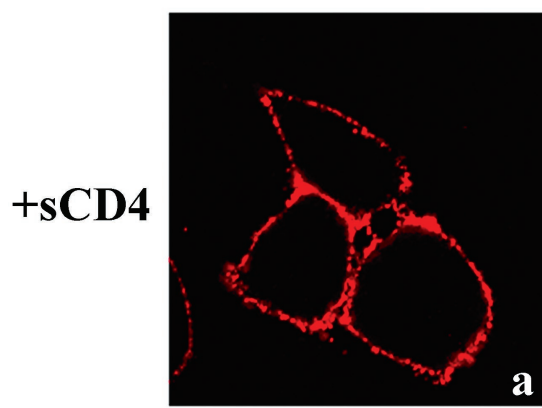

a

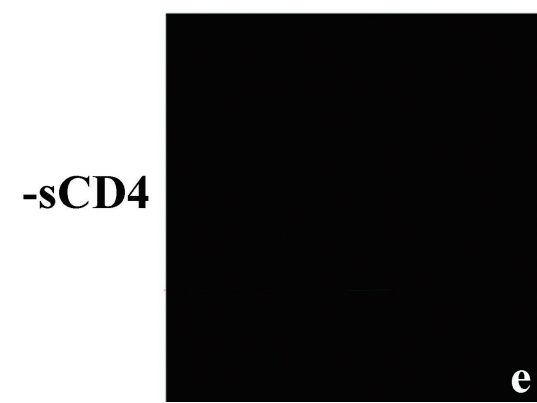

48d
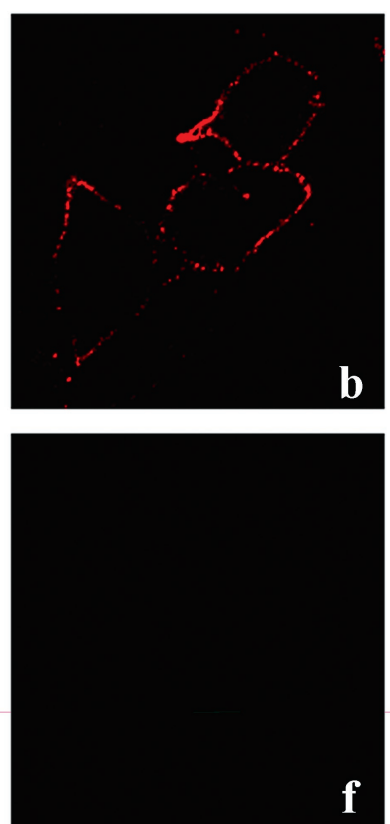

CG10
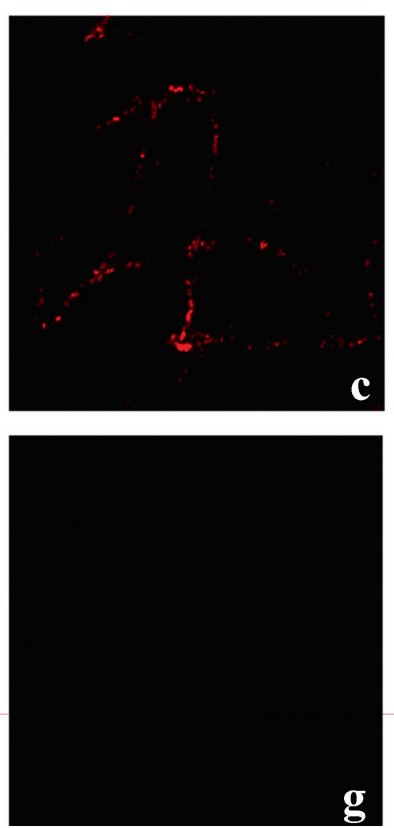

$17 b$ (unfixed cells)
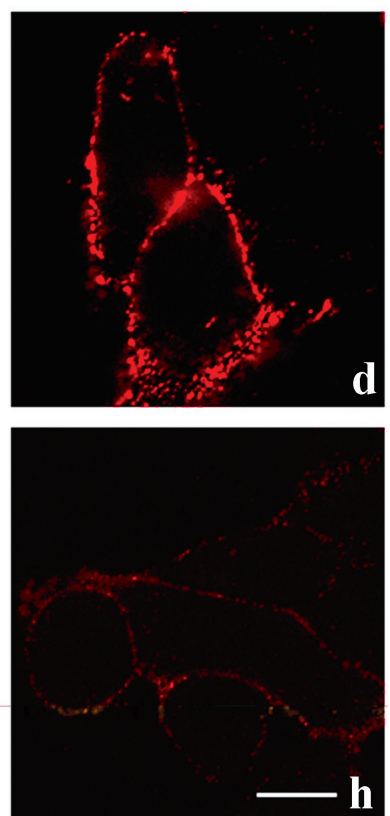

FIG. 2. MAbs 17b, 48d, and CG10 bind to HIV envelope-expressing cells in the presence of sCD4. Env cells were either untreated or incubated with $1 \mu \mathrm{g}$ of sCD4/ml in DMEM for $120 \mathrm{~min}$ at $4^{\circ} \mathrm{C}$ and then either fixed with $\mathrm{BS}^{3}$ or directly stained with the indicated MAbs (visible in red) as described in Materials and Methods. MAbs were tested in parallel, along with human isotype controls, which produced no binding signal (data not shown). Representative images are shown. Each experiment was repeated at least three times with the same results. Scale bar, $10 \mu \mathrm{m}$.

transition of gp41 into helix bundles (19) during cell-cell fusion. The latter method covalently cross-links and immobilizes surface proteins within 1 min (data not shown) without membrane permeabilization and loss of dye. Cocultured Env and target cells arrested within $10 \mathrm{~min}$ at $37^{\circ} \mathrm{C}$ by either method were clearly attached but did not yet exhibit cytoplasmic dye transfer (Fig. 1). In accordance with previous studies (10, 19, 29), arrested cells exhibited signs of fusion after $30 \mathrm{~min}$ at $37^{\circ} \mathrm{C}$ marked by the appearance of clusters diffusely stained as a result of cytoplasmic dye transfer (Fig. 1). The number of fusion events accumulated over time such that by $120 \mathrm{~min}$ most fields contained large, diffusely staining cell clusters (syncytia) with multiple areas of nuclear dye exclusion.

Exposure of the coreceptor binding domain on gp120 during cell-cell fusion. In order to examine the exposure of antigenic domains on intermediate gp120 structures during syncytium formation, the arrested cells were immunostained with selected MAbs. In one series of experiments we attempted to characterize antigenic changes in the CD4-induced coreceptorbinding site on gp120 by using the anti-coreceptor binding domain MAbs 17b, 48d, and CG10 (22, 27, 33). As shown in Fig. 1, MAb reactivity was only observed on Env cells arrested during the fusion process by chilling to $4^{\circ} \mathrm{C}$. Representative fields of digitally enhanced $17 \mathrm{~b}$ binding signals are shown; similar but somewhat fainter staining was obtained with $48 \mathrm{~d}$ and CG10 (data not shown). In every case, MAb reactivity was generally distributed (Fig. 1) and apparent before cytoplasmic dye transfer, even on areas of Env cells not in contact with target cells (Fig. 1, arrows). Such binding appeared to be specific since no signal was detected with an isotype control antibody tested under identical staining and imaging conditions (data not shown). However, there was no evidence of locally intensified MAb staining at interfaces of attached Env and target cells (Fig. 1) where gp120-CD4 complexes form. Instead, lower binding signals were often evident at cell attachment sites in many fields. Notably, none of the MAbs reacted with cells arrested by $\mathrm{BS}^{3}$ cross-linking (Fig. 1). No detectable binding occurred on any portion of attached Env cells not yet undergoing fusion (Fig. 1; 10 and $20 \mathrm{~min}$ ) or on fusing Env cells arrested at 30,60, or $120 \mathrm{~min}$ after coculture with target cells. The same results were obtained with cells arrested and fixed with PFA prior to antibody staining (data not shown), indicating that the absence of MAb binding was not specifically related to $\mathrm{BS}^{3}$ cross-linking.

These results contrasted to what was observed with Env cells after treatment with sCD 4 for $120 \mathrm{~min}$ at $4^{\circ} \mathrm{C}$. As shown in Fig. 2 , such treatment markedly increased the binding signal with either $\mathrm{BS}^{3}$-fixed or cooled, unfixed Env cells as a result of gp120-CD4 complex formation and enhanced exposure of the coreceptor-binding domain. MAb $17 \mathrm{~b}$ exhibited the greatest increase in staining after sCD4 treatment; the changes observed with $48 \mathrm{~d}$ or CG10 were much less pronounced (data not shown). To verify these results, the assays were repeated by using a europium-tagged secondary antibody that allowed quantitative detection of total $17 \mathrm{~b}$ binding in an entire assay well. In agreement with the microscopy experiments, $17 \mathrm{~b}$ did not produce a signal above that obtained with the isotype control except when the Env cells were treated with sCD4 (data not shown). Therefore, our inability to detect intensified MAb staining specifically at Env-target cell interfaces suggested that the coreceptor-binding domain might be blocked during cell-cell fusion. 


\section{$17 b$}

\section{fixed cells unfixed cells}
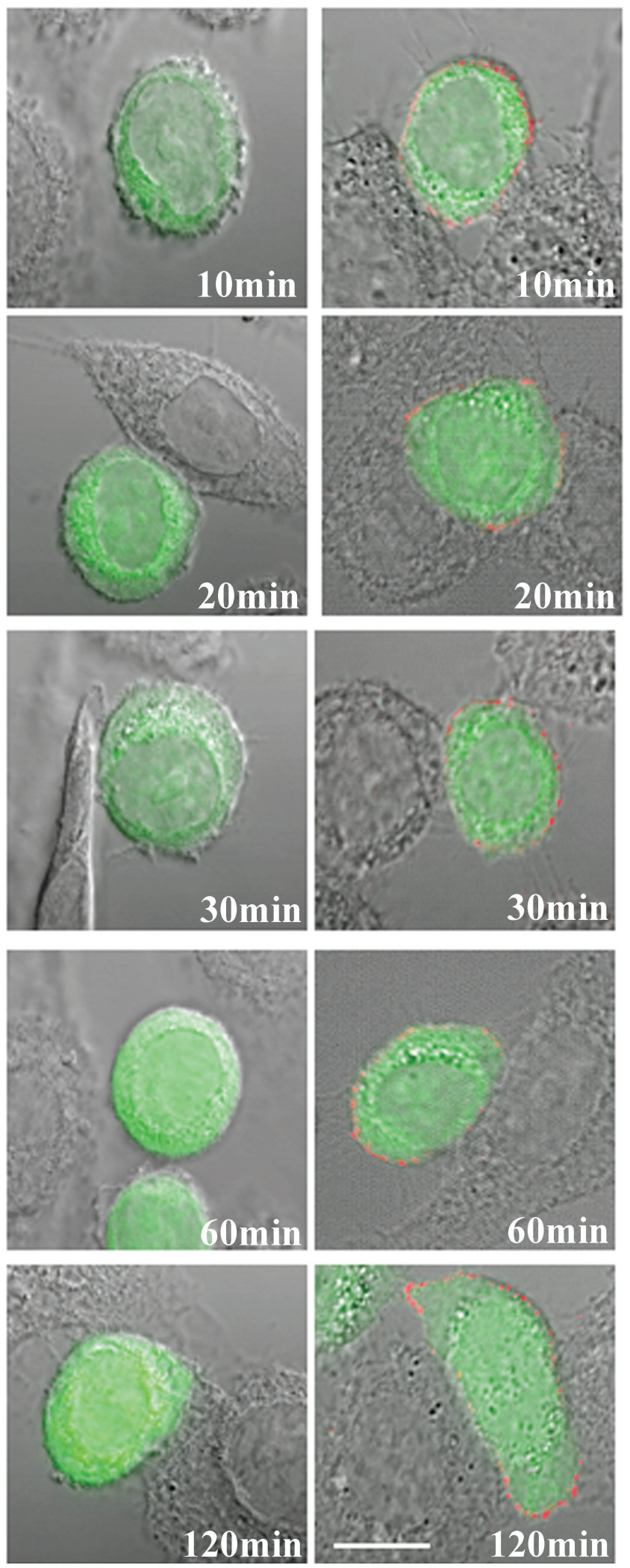

To address this possibility, we examined whether MAb binding at the Env-target cell interface might be competitively blocked by interactions of the coreceptor-binding site with CXCR4. The assays were repeated, this time after treatment of the target cells with SDF-1 to prevent envelope interactions with CXCR4 (26). Analyses of the SDF-treated cells by immunofluorescence and flow cytometry confirmed that CXCR4 was down regulated on the target cells, whereas CD4 expression levels remained unaltered (data not shown). As shown in Fig. 3 , SDF-1 clearly inhibited cell-cell fusion, as evidenced by the absence of apparent dye transfer or syncytium formation over a 10- to 120-min cocultivation period. However, 48d and CG10 binding (data not shown) or $17 \mathrm{~b}$ binding (Fig. 3) was not altered or intensified as a result of chemokine treatment, indicating that the MAbs were not being blocked by coreceptor interactions at the Env-target cell interface.

Therefore, we examined the alternative possibility that MAb binding was occluded by physical restrictions inherent in the orientation of interacting membranes at the Env-target cell interface. In this case, it might be possible to obtain better immunostaining by using a smaller $17 \mathrm{~b}$ Fab fragment. However, like the intact MAbs a $17 \mathrm{~b}$ Fab exhibited no detectable binding to $\mathrm{BS}^{3}$-fixed cells (data not shown) at any time during the fusion process and only faint, generalized reactivity with the cooled and unfixed cells. There was no evidence of greater Fab binding at Env-target cell interfaces (data not shown); the signal was the same as that obtained with the intact 17b MAb. Further evidence that the coreceptor-binding domain was not accessible to the Fab at the interface of unfixed cells was provided by assays to detect MAb neutralization of cell-cell fusion. As shown in Fig. 4, up to $100 \mu \mathrm{g}$ of either 17b Fab or intact $\mathrm{MAbs} / \mathrm{ml}$ did not neutralize cell-cell fusion versus control normal human immunoglobulin (Fig. 4), whereas the expected strong inhibition was seen with a broadly neutralizing antibody, MAb 2G12 (28). The 17b Fab also failed to demonstrate stronger reactivity with Env cell membranes in contact with CXCR4-negative U373 cells expressing only CD4 (data not shown). However, the $17 \mathrm{~b}$ Fab demonstrated markedly enhanced binding to sCD4-treated Env cells (data not shown) similar to what was seen with the intact $17 \mathrm{~b}$. Thus, the coreceptor-binding domain appeared to be blocked at the cell-cell interface in a manner that does not require coreceptor interactions and cannot be overcome by smaller Fab molecules.

Exposure of CD4-induced epitopes outside the coreceptorbinding domain during cell-cell fusion. We next attempted to characterize fusion-dependent antigenic changes in a different gp120 domain that is selectively presented within the C1-C4/C5 region of soluble gp120 after binding to $\mathrm{CD} 4(21,34)$. A mu-

FIG. 3. SDF-1 blocks cell-cell fusion but does not enable $17 \mathrm{~b}$ binding. CellTracker Green-labeled Env cells and target cells were pretreated with $3 \mu \mathrm{g}$ of SDF- $1 / \mathrm{ml}$ for $1 \mathrm{~h}$ at $37^{\circ} \mathrm{C}$ prior to cocultivation. The cells were then incubated for the indicated times as described in Materials and Methods in the presence of $3 \mu \mathrm{g}$ of SDF-1/ml. Fusing cells were either fixed with $\mathrm{BS}^{3}$ or cooled to $4^{\circ} \mathrm{C}$ and immunostained with $17 \mathrm{~b}$ at $5 \mu \mathrm{g} / \mathrm{ml}$. Reactivity was detected only with the unfixed cells; due to the faint signal, the images were digitally enhanced for clarity. Representative images are shown. Each experiment was repeated at least three times with the same results. Scale bar, $10 \mu \mathrm{m}$. 


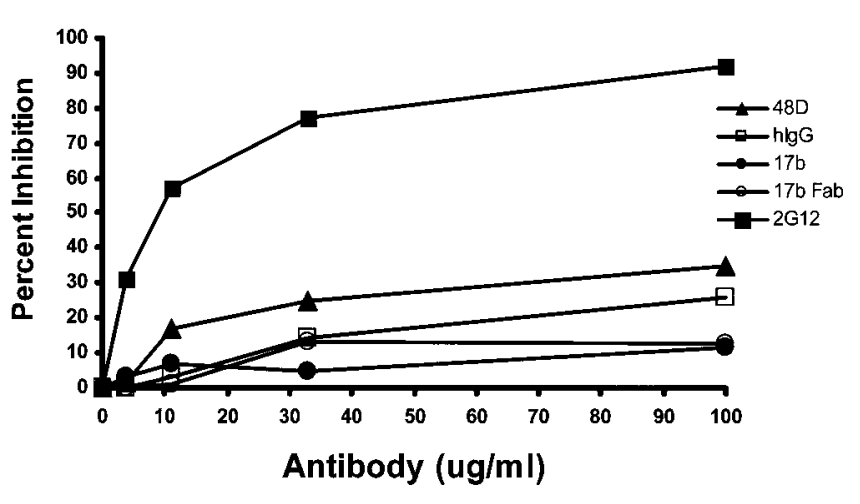

FIG. 4. 17b Fab and MAbs directed against the gp120 coreceptorbinding site do not inhibit HIV-mediated cell-cell fusion. Neutralization activity was assayed by using Env and target cells as described in Materials and Methods. Env cells $\left(10^{4}\right)$ were preincubated in triplicate wells with threefold serial dilutions of the indicated MAbs or Fab, starting at $100 \mu \mathrm{g} / \mathrm{ml}$. The treated Env cells were then added to $10^{4}$ target cells. Negative control assays were carried out in the absence of antibody or with normal human IgG (hIgG); positive control experiments included the broadly neutralizing MAb 2G12 (21). Cell-cell infection was determined after incubation at $37^{\circ} \mathrm{C}$ for $18 \mathrm{~h}$ by quantitative $\beta$-galactosidase assay. The percent inhibition of fusion for each test condition was calculated relative to control assays carried out in the absence of antibody. The averages of triplicate assays are shown.

rine MAb, 8F101 (5), and a human MAb, A32 (21, 34), that recognize related epitopes in this domain (J. Binley, unpublished data) were selected for the analyses. As shown in Fig. 5, staining with these MAbs was not observed on interactive Env and target cells for the first $20 \mathrm{~min}$ of cocultivation. However, surface reactivity was evident after $30 \mathrm{~min}$ of coculture, coincident with the initial transfer of cytoplasmic dye (Fig. 5). Staining (visible in red) was observed on cells exhibiting cytoplasmic mixing and typically surrounded the fused cell membranes. More notably, MAb staining was observed on some clusters of cells that had not yet fused or exhibited cytoplasmic mixing. In these cases reactivity was localized to small patches at the Env-target cell interfaces (Fig. 5, arrows). At later times (60 and $120 \mathrm{~min}$ ), multiple centers of multinucleated cells exhibiting diffuse cytoplasmic staining were more intensely reactive with both MAbs. Identical binding patterns were observed with either MAb when fusion intermediates were arrested by rapid chilling to $4^{\circ} \mathrm{C}$ rather than by cross-linking (data not shown). No such binding was observed with the other MAbs (Fig. 1) or with murine and human isotype controls (data not shown).

In control experiments, the MAbs reacted strongly with CD4-expressing cells treated with soluble gp120 (data not shown) in agreement with their ability to recognize soluble gp120-CD4 complexes $(6,9,35)$. However, the MAbs did not react with Env cells treated for up to $120 \mathrm{~min}$ with either sCD4or CXCR4-negative U373 cells expressing only CD4 (data not shown). Thus, the conditions for MAb binding to membraneanchored HIV envelope, unlike soluble gp120, seemed dependent on the presence of both CD4 and CXCR4 on the target cell.

To investigate this possibility further, the fusion experiment was repeated with target cells treated with SDF-1 to selectively block coreceptor interactions. As shown in Fig. 6, SDF treat- ment inhibited fusion and cytoplasmic dye transfer and at the same time eliminated 8F101 staining of cross-linked cells arrested in coculture over a period of $120 \mathrm{~min}$ (Fig. 6B). Identical results were obtained with A32 with SDF-1-treated cells (data not shown) and in experiments with unfixed cells arrested by cooling them to $4^{\circ} \mathrm{C}$ (data not shown). In comparison, treatment of the coculture with a fusion inhibitor, T21 (32), that targets gp41 prevented dye transfer but allowed MAb binding to the arrested cells. Notably, such binding was exclusively restricted to patches at the Env-target cell interface (Fig. 6A), similar to what was observed after $30 \mathrm{~min}$ of coculture (Fig. 5). Such patches persisted for at least $120 \mathrm{~min}$ of coculture. These experiments further suggested that MAb binding to the fusing cells was dependent on membrane-anchored envelope interactions with coreceptor and the formation of surface gp120-CD4coreceptor tricomplexes.

However, since the Env cells theoretically had the capacity to shed gp120, it was possible that the staining pattern seen over the syncytium surfaces at later times was due in part to the cumulative binding of free envelope shed into the culture fluid. To address this possibility, the target cells were incubated for $120 \mathrm{~min}$ at $37^{\circ} \mathrm{C}$ with conditioned media collected from a $2-\mathrm{h}$ culture of Env cells maintained at the same density used in the fusion assay. The treated cells were then analyzed with A32 and 8F101 as before. Under these conditions, no MAb binding was detected (data not shown), illustrating that any gp120 that might have been shed into the surrounding medium was not responsible for the observed staining pattern.

\section{DISCUSSION}

Virus-cell fusion in HIV infection involves a series of intermediate structures formed between the HIV envelope and cell surface receptors. These intermediates have been proposed as immunogens to elicit fusion-blocking humoral responses (5, 17) and in theory could serve as targets for chemotherapeutic agents. Complexes of soluble molecules, or their derivatives, have been useful in examining epitope exposure on HIV envelope intermediates $(6,27)$; however, several studies have suggested that such antigens may not directly reflect or predict what epitopes are expressed or accessible on the surfaces of fusing cells $(23,25)$.

In this study, we analyzed the exposure of epitopes on gp120 intermediates during the progression of syncytium formation. In accordance with previous reports $(10,19,29)$, we detected the initiation of cell-cell fusion in our system after $30 \mathrm{~min}$ at $37^{\circ} \mathrm{C}$, as indicated by the diffusion of dye from the envelope expressing cells into the cytoplasm of the target cells. The formation of syncytia then progressed over the next $2 \mathrm{~h}$, producing multiple centers of multinucleated cells with diffuse cytoplasmic staining. Using a fixable dye to visualize the fusion process, we were able to arrest the cellular intermediates at various time points and stain them with antibodies directed against receptor-induced domains on gp120. This provided a unique view of epitope exposure on the surfaces of cells undergoing active fusion.

Our analyses with MAbs 17b, 48d, and CG10 indicated that the coreceptor-binding domain exhibits a limited but constitutive exposure on envelope expressing cells, in agreement with previous studies $(3,24)$. MAb binding, albeit faint, was gener- 
8F101
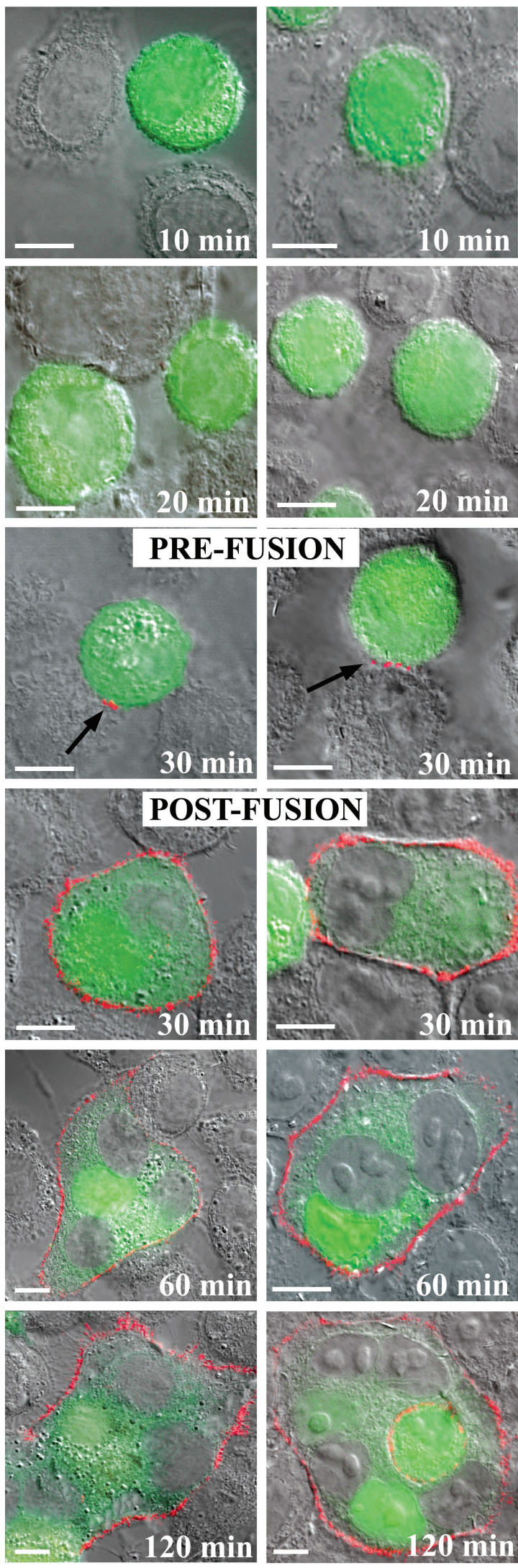

A32
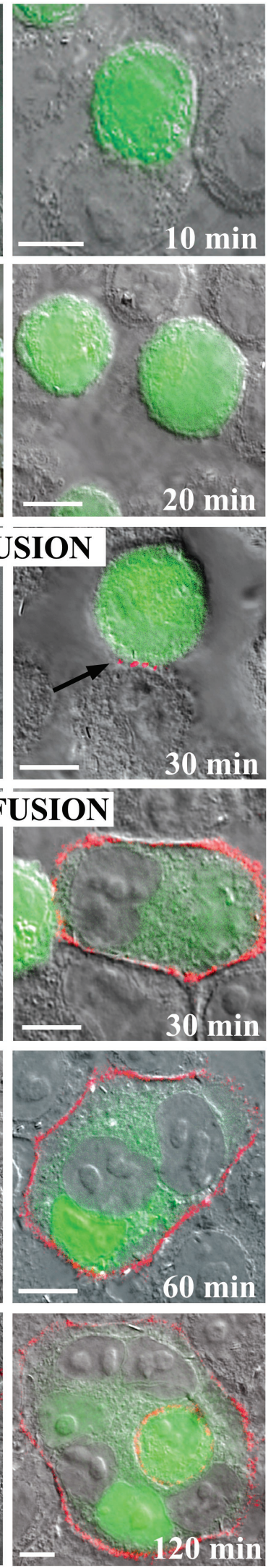

ally distributed over the Env cell surfaces but was independent of contact with $\mathrm{CD}^{+}{ }^{+}$target cells (Fig. 1, arrows) or exposure to sCD4 (Fig. 2). Notably, such reactivity was only obtained with unfixed cells and was not seen when surface proteins were cross-linked with $\mathrm{BS}^{3}$ (Fig. 1) or fixed with PFA (data not shown). Such differences indicate that some amount of flexibility in the envelope structure may either permit constitutive exposure of the coreceptor-binding domain without CD4 engagement or otherwise facilitate cognate antibody binding. The factors allowing MAb binding to temperature-arrested versus fixed envelope are being investigated.

As expected, we were able to clearly increase the exposure of the coreceptor-binding domain by treating Env cells with sCD4 (Fig. 2). It was therefore noteworthy that we did not observe enhanced MAb binding as a result of interactions between Env and $\mathrm{CD}^{+}{ }^{+}$target cells (Fig. 1). There was no evidence of enhanced or selective $17 \mathrm{~b}, 48 \mathrm{~d}$, and CG10 MAb binding to the unfixed Env-target cell interface at $4^{\circ} \mathrm{C}$ and no apparent reactivity with cocultured cells fixed by covalent cross-linking. Further, the MAbs completely failed to block fusion of the Env and target cells (Fig. 4). Taken together, these findings are consistent with a model, suggested by previous studies (7, 20, 23 ), in which the coreceptor binding site remains largely occluded from cognate antibodies as it becomes induced at the cell-cell fusion interface.

Notably, competitive coreceptor interactions with the coreceptor-binding domain do not appear to be responsible for obstructing the MAb binding in our experiments. Treatment of the CD4- and CXCR4-expressing target cells with SDF-1 failed to promote $17 \mathrm{~b}, 48 \mathrm{~d}$, or CG10 binding to the Env-target cell interface (Fig. 3 and data not shown) and yet clearly blocked fusion. Notably, all of the antibodies reacted with Env cells after treatment with sCD4 (Fig. 2) and with target cells coated with soluble envelope (data not shown) in agreement with an earlier report (25). These findings introduce the possibility that the close physical proximity of fusing cell membranes, rather than coreceptor interactions, restrict MAb binding. However, such a restriction may be relatively severe given that the binding characteristics we observed with smaller $17 \mathrm{~b}$ Fab fragments versus intact antibody were essentially the same. In any case, such a scenario is consistent with, and provides an explanation for, previous demonstrations that antibodies such

FIG. 5. Temporal expression of the 8F101 and A32 epitopes on gp120 during cell-cell fusion. CellTracker Green-labeled Env cells were cocultivated with target cells for the indicated times as described in Materials and Methods. Immunostaining was performed with $\mathrm{BS}^{3}$ fixed cells as described in the text by using the MAbs 8F101 and A32 (visible in red) at 5 and $1 \mu \mathrm{g} / \mathrm{ml}$, respectively. Two images are shown for each MAb at $30 \mathrm{~min}$. One image depicts Env cells that have not yet undergone fusion, as indicated by the lack of cytoplasmic dye transfer. The second image depicts the MAb staining pattern observed after cell-cell fusion. Arrows indicates patches of 8F101 and A32 staining on unfused cells present 30 min after cocultivation. MAbs were tested in parallel, along with human and murine isotype controls. Isotype controls produced no binding signal (data not shown). Representative images are shown. Each experiment was repeated at least three separate times with the same results. Scale bar, $10 \mu \mathrm{m}$. In contrast to $17 \mathrm{~b}$, 48d, and CG10 (Fig. 1), the relatively strong binding signals obtained with these antibodies did not require digital enhancement. 

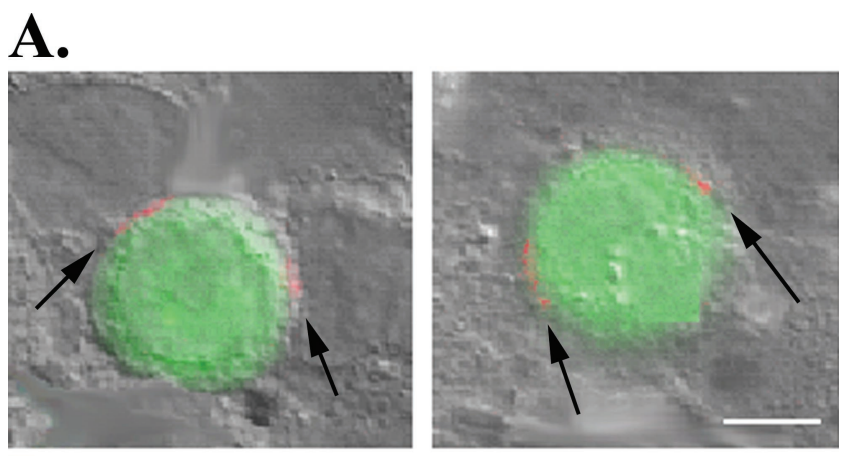

\section{B.}
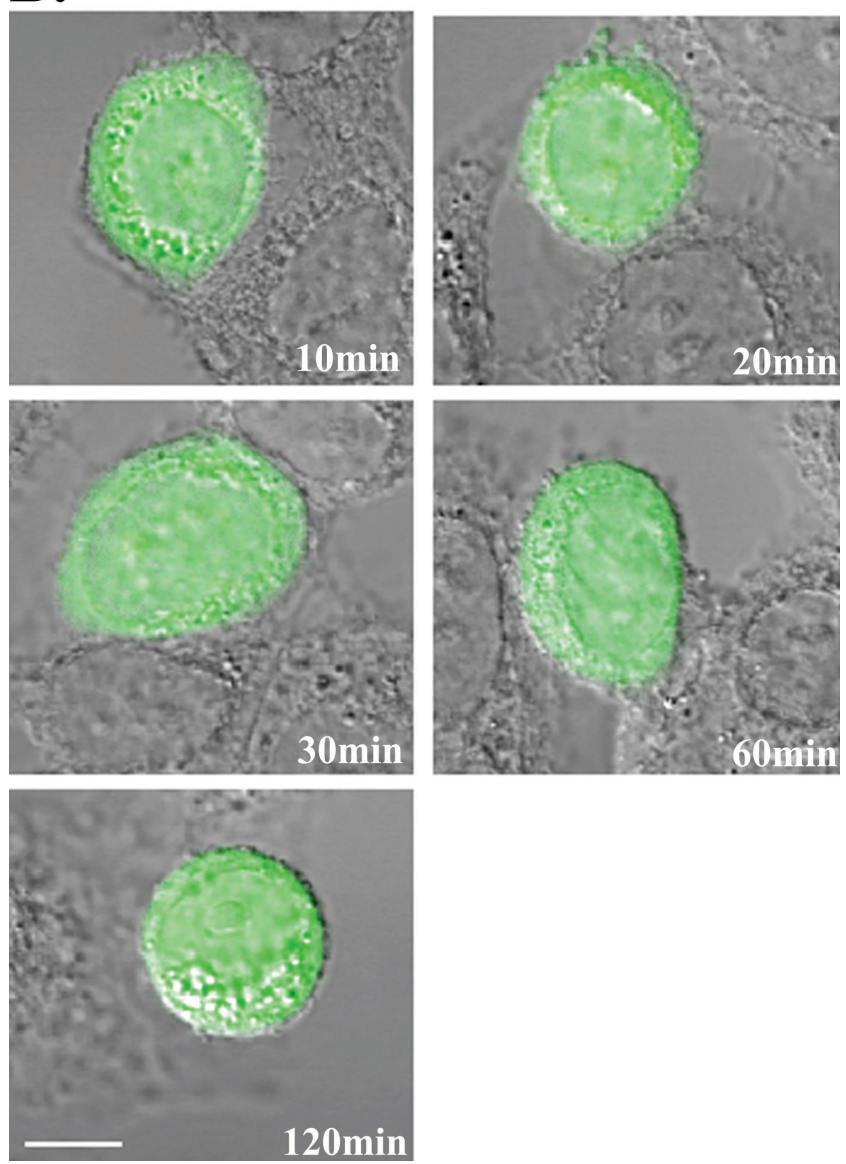

FIG. 6. Effects of fusion inhibitors on 8F101 epitope exposure. (A) CellTracker Green-labeled Env cells and target cells were cocultivated for $120 \mathrm{~min}$ in the presence of $5 \mu \mathrm{g}$ of $\mathrm{T} 21 / \mathrm{ml}$. Interacting cells were fixed with $\mathrm{BS}^{3}$ and immunostained with $8 \mathrm{~F} 101$ at $5 \mu \mathrm{g} / \mathrm{ml}$. Arrows indicate patches of MAb staining (visible in red) on the cell-cell interface. Representative images are shown. Each experiment was repeated at least three times with the same results. Scale bar, $10 \mu \mathrm{m}$. (B) CellTracker Green-labeled Env cells and target cells were pretreated with $3 \mu \mathrm{g}$ of SDF- $1 / \mathrm{ml}$ for $1 \mathrm{~h}$ at $37^{\circ} \mathrm{C}$ prior to cocultivation. The cells were then incubated for the indicated times as described in Materials and Methods in the presence of $3 \mu \mathrm{g}$ of SDF-1/ml. Interacting cells were fixed with $\mathrm{BS}^{3}$ and immunostained with $8 \mathrm{~F} 101$ at $5 \mu \mathrm{g} / \mathrm{ml}$. Representative images are shown. Each experiment was repeated at least three times with the same results. Scale bar, $10 \mu \mathrm{m}$. as $17 \mathrm{~b}$ are poorly neutralizing except in instances where fusion is facilitated by sCD4 (23).

Another explanation for our inability to detect localized 17b, $48 \mathrm{~d}$, or CG10 MAb binding at the Env-target cell interface is that the gp120-CD4 complexes being formed at any time during fusion are simply too rare to allow detection by microscopy. However, the inability of the antibodies to neutralize cell-cell fusion (Fig. 4) argues against this possibility; antibody recognition of limited yet essential structures should have yielded strong neutralization rather than the observed null effect. Furthermore, we were able to specifically stain Env-target cell interfaces with the other MAbs (8F101 and A32) even at early time points, indicating that HIV envelope intermediates are abundant enough to be detected during the fusion process.

In contrast to the 17b-like antibodies, MAbs 8F101 and A32, which bind a CD4-induced domain outside the coreceptorbinding site, produced clearly discernible staining that changed over time in concert with the progression of fusion. Although these MAbs recognize soluble gp120-CD4 complexes $(6,9,35)$, three lines of evidence suggested that staining at the cell surface was entirely dependent on the formation of gp120-CD4coreceptor tricomplex. First, MAb binding became apparent only after $30 \mathrm{~min}$ of coculture and corresponded with the appearance of cells exhibiting cytoplasmic dye transfer. At this time, a number of interacting Env and target cells did not yet demonstrate cytoplasmic mixing but were stained by the MAbs at specific points located at the Env-target cell interface (Fig. $5)$. Second, the antibodies failed to react with cocultured cells treated with SDF-1 (Fig. 6B) but bound to the Env-target cell interface in the presence of a fusion inhibitor specific for gp41 (Fig. 6A). Third, the MAbs stained cells expressing CD4 or CD4 and CXCR4 after treatment with soluble gp120 but failed to react with Env cells treated with sCD4 (data not shown).

This apparent dependence on tricomplex formation is most likely explained by the characteristics of the 8F101/A32-binding domain in gp120. Previous studies have placed this CD4induced domain $(9,35)$ in the $\mathrm{C} 1-\mathrm{C} 4 / \mathrm{C} 5$ region of gp120 (21, $34)$, which is ordinarily blocked by gp41 on membrane-anchored HIV envelope (13). Therefore, the induced 8F101 and A32 domain should only be exposed in the absence of an interaction with gp41. In accordance with this, the MAbs are known to bind to soluble gp120-CD4 complexes $(6,9,35)$ and in these studies bound to target cells treated with soluble gp120 (data not shown). However, in the context of cell-cell fusion the MAbs are almost certainly specific for the gp120-CD4coreceptor tricomplex, since this is the only intermediate envelope structure that can dissociate from gp41 and yet remain bound to a fusing cell membrane. Our findings verify that the exposure of these epitopes and coincident MAb binding occurs, as expected, in concert with the initial signs of cell-cell membrane fusion and cytoplasmic mixing.

The staining patterns exhibited by MAbs 8F101 and A32 provide important insights into our understanding of the fate of tricomplexes during the course of cell-cell HIV infection. An examination of MAb binding to cells arrested after extended times in coculture indicated that tricomplexes accumulate and disperse over the surface of the syncytium membrane over a period of at least $2 \mathrm{~h}$. This staining did not reflect cumulative binding of shed gp120, since treating target cells with conditioned medium collected from the envelope-express- 
ing cells after $2 \mathrm{~h}$ in culture did not produce discernible staining (data not shown). Instead, the tricomplexes are probably formed continuously as the cell membranes fuse and disperse into the syncytium membrane over time. Our ability to detect an 8F101 signal over a 2-h period is consistent with recent studies (16) indicating that the half-life of gp120-CD4-coreceptor tricomplexes is 1 to $2 \mathrm{~h}$. Thus, in the time frame of our assay many of the tricomplexes would have remained on the cell surface rather than becoming internalized. This persistence may have important implications for modulating and maintaining a specific host cell environment given the recent demonstration of HIV envelope-mediated intracellular signaling via coreceptor interactions (31).

Collectively, our data have two important implications for HIV vaccine development. First, the apparent inability of $17 \mathrm{~b}$, 48d, and CG10 to recognize gp120-CD4 complexes forming at the fusing cell interface indicates that neutralizing humoral responses to the coreceptor-binding site may be extremely difficult, if not impossible, to obtain. An antigen capable of placing the coreceptor-binding site in an immunogenic context would likely elicit antibodies that are unable to bind the critical epitope at the fusing cell interface. Therefore, efforts to inhibit HIV entry with humoral responses may need to place greater emphasis on neutralizing determinants on gp120-CD4 complexes, or perhaps even gp120-CD4-coreceptor tricomplexes, that lie outside the coreceptor-binding domain. This approach seems theoretically possible, since our results indicate that intermediate envelope structures can be accessed at the fusing cell interface. Second, the pattern of 8F101 and A32 emphasizes that the critical gp120 intermediates governing viral entry are relatively rare during the initial stages of cell-cell fusion. Therefore, strategies to elicit humoral responses against certain key envelope intermediates through the use of fixed-cell, or "fusion-competent," immunogens (17) may be problematic, since the effective concentrations of desired immunogen are likely to be extremely low in most formulations. Soluble immunogens designed to closely mimic key HIV surface structures may provide more feasible vaccine candidates. In any case, the design of such immunogens will have to carefully consider whether the presentation of epitopes is consistent with what occurs in the context of cell-cell infection.

\section{ACKNOWLEDGMENTS}

We thank James Robinson, Tulane University, New Orleans, La., for kindly providing the human MAbs 17b, A32, and 48d; Jonathan Gershoni, Tel Aviv University, for his gift of MAb CG10; Ranajit Pal, Advanced BioScience Laboratories, Kensington, Md., for providing the 8F101 hybridoma; Hermann Katinger of IAM Pharmaceuticals, Inc., Vienna, Austria, for supplying MAb 2G12; and Werner Meier for his gift of sCD4. We also thank Timothy Fouts for helpful discussions.

This work was supported in part by grants NHLBI R01 03-5-20064, R21 03-5-21326, and PO1 03-5-21332 to A.L.D.

\section{REFERENCES}

1. Alkhatib, G., C. Combadiere, C. C. Broder, Y. Feng, P. E. Kennedy, P. M. Murphy, and E. A. Berger. 1996. CC CKR5: a RANTES, MIP-1 $\alpha$, MIP-1 $\beta$ receptor as a fusion cofactor for macrophage-tropic HIV-1. Science 272: 1955-1958.

2. Berger, E. A., P. M. Murphy, and J. M. Farber. 1999. Chemokine receptors as HIV-1 coreceptors: roles in viral entry, tropism, and disease. Annu. Rev. Immunol. 17:657-700.

3. Binley, J. M., R. W. Sanders, B. Clas, N. Schuelke, A. Master, Y. Guo, F. Kajumo, D. J. Anselma, P. J. Maddon, W. C. Olson, and J. P. Moore. 2000 A recombinant human immunodeficiency virus type 1 envelope glycoprotein complex stabilized by an intermolecular disulfide bond between the gp 120 and gp41 subunits is an antigenic mimic of the trimeric virion-associated structure. J. Virol. 74:627-643.

4. Chan, D. C., and P. S. Kim. 1998. HIV entry and its inhibition. Cell 93:681684.

5. DeVico, A., A. Silver, A. M. Thornton, M. G. Sarngadharan, and R. Pal. 1996. Covalently crosslinked complexes of human immunodeficiency virus type 1 gp120 and CD4 receptor elicit a neutralizing immune response that includes antibodies selective for primary virus isolates. Virology 218:258263.

6. DeVico, A. L., R. Rahman, J. Welch, R. Crowley, P. Lusso, M. G. Sarngadharan, and R. Pal. 1995. Monoclonal antibodies against covalently cross-linked complexes of human immunodeficiency virus type $1 \mathrm{gp} 120$ and CD4 receptor identify a novel complex-dependent epitope on gp120. Virology 211:583588.

7. D'Souza, M. P., G. Milman, J. A. Bradac, D. McPhee, C. V. Hanson, and R. M. Hendry. 1995. Neutralization of primary HIV-1 isolates by anti-envelope monoclonal antibodies. AIDS 9:867-874.

8. Feng, F., C. C. Broder, P. E. Kennedy, and E. A. Berger. 1996. HIV-1 entry cofactor: functional cDNA cloning of a seven transmembrane, G proteincoupled receptor. Science 272:872-877.

9. Fouts, T. R., J. M. Binley, A. Trkola, J. E. Robinson, and J. P. Moore. 1997. Neutralization of the human immunodeficiency virus type 1 primary isolate JR-FL by human monoclonal antibodies correlates with antibody binding to the oligomeric form of the envelope glycoprotein complex. J. Virol. 71:27792785.

10. Frey, S., M. Marsh, S. Gunther, A. Pelchen-Matthews, P. Stephens, S. Ortlepp, and T. Stegmann. 1995. Temperature dependence of cell-cell fusion induced by the envelope glycoprotein of human immunodeficiency virus type 1. J. Virol. 69:1462-1472.

11. Fu, Y. K., T. K. Hart, Z. L. Jonak, and P. J. Bugelski. 1993. Physicochemical dissociation of CD4-mediated syncytium formation and shedding of human immunodeficiency virus type 1 gp120. J. Virol. 67:3818-3825.

12. Hart, T. K., A. Truneh, and P. J. Bugelski. 1996. Characterization of CD4gp120 activation intermediates during human immunodeficiency virus type 1 syncytium formation. AIDS Res. Hum. Retrovir. 12:1305-1313.

13. Helseth, E., U. Olshevsky, C. Furman, and J. Sodroski. 1991. Human immunodeficiency virus type 1 gp120 envelope glycoprotein regions important for association with the gp41 transmembrane glycoprotein. J. Virol. 65:21192123.

14. Jernigan, K. M., R. Blumenthal, and A. Puri. 2000. Various effects of temperature, $\mathrm{Ca}^{2+}$, and cytochalasin on fusion activity mediated by human immunodeficiency virus type 1 and type 2 glycoproteins. FEBS Lett. 474: 246-251.

15. Kimpton, J., and M. Emerman. 1992. Detection of replication-competent and pseudotyped human immunodeficiency virus with a sensitive cell line on the basis of activation of an integrated $\beta$-galactosidase gene. J. Virol. 66: 2232-2239.

16. Kozak, S. L., S. E. Kuhmann, E. J. Platt, and D. Kabat. 1999. Roles of CD4 and coreceptors in binding, endocytosis, and proteolysis of gp120 envelope glycoproteins derived from human immunodeficiency virus type 1. J. Biol. Chem. 274:23499-23507.

17. LaCasse, R. A., K. E. Follis, M. Trahey, J. D. Scarborough, D. R. Littman, and J. H. Nunberg. 1999. Fusion-competent vaccines: broad neutralization of primary isolates of HIV. Science 283:357-362.

18. Lee, S., K. Peden, D. S. Dimitrov, C. C. Broder, J. Manischewitz, G. Denisova, J. M. Gershoni, and H. Golding. 1997. Enhancement of human immunodeficiency virus type 1 envelope-mediated fusion by a CD4-gp120 complex-specific monoclonal antibody. J. Virol. 71:6037-6043.

19. Melikyan, G. B., R. M. Markosyan, H. Hemmati, M. K. Delmedico, D. M. Lambert, and F. S. Cohen. 2000. Evidence that the transition of HIV-1 gp41 into a six-helix bundle, not the bundle configuration, induces membrane fusion. Cell Biol. 151:413-424.

20. Moore, J. P., F. E. McCutchan, S. W. Poon, J. Mascola, J. Liu, Y. Cao, and D. D. Ho. 1994. Exploration of antigenic variation in gp120 from clades A through $\mathrm{F}$ of human immunodeficiency virus type 1 by using monoclonal antibodies. J. Virol. 68:8350-8364.

21. Moore, J. P., and J. Sodroski. 1996. Antibody cross-competition analysis of the human immunodeficiency virus type 1 gp120 exterior envelope glycoprotein. J. Virol. 70:1863-1872.

22. Rizzuto, C. D., R. Wyatt, N. Hernandez-Ramos, Y. Sun, P. D. Kwong, W. A. Hendrickson, and J. Sodroski. 1998. A conserved HIV gp120 glycoprotein structure involved in chemokine receptor binding. Science 280:1763-1767.

23. Salzwedel, K., E. D. Smith, B. Dey, and E. A. Berger. 2000. Sequential CD4-coreceptor interactions in human immunodeficiency virus type 1 Env function: soluble CD4 activates Env for coreceptor-dependent fusion and reveals blocking activities of antibodies against cryptic conserved epitopes on gp120. J. Virol. 74:326-333.

24. Sattentau, Q. J., and J. P. Moore. 1995. Human immunodeficiency virus type 1 neutralization is determined by epitope exposure on the gp120 oligomer. J. Exp. Med. 182:185-196.

25. Sullivan, N., Y. Sun, Q. Sattentau, M. Thali, D. Wu, G. Denisova, J. Ger- 
shoni, J. Robinson, J. Moore, and J. Sodroski. 1998. CD4-induced conformational changes in the human immunodeficiency virus type $1 \mathrm{gp} 120$ glycoprotein: consequences for virus entry and neutralization. J. Virol. 72:46944703 .

26. Tarasova, N. I., R. H. Stauber, and C. J. Michejda. 1998. Spontaneous and ligand-induced trafficking of CXC-chemokine receptor 4. J. Biol. Chem. 273:15883-15886.

27. Thali, M., J. P. Moore, C. Furman, C. McArthur, D. D. Ho, J. Robinson, and J. Sodroski. 1993. Characterization of conserved human immunodeficiency virus type 1 gp120 neutralising epitopes exposed on gp120-CD4 binding. J. Virol. 67:3978-3988.

28. Trkola, A., A. B. Pomales, H. Yuan, B. Korber, P. J. Maddon, G. P. Allaway, H. Katinger, C. F. Barbas III, D. R. Burton, D. D. Ho, and J. P. Moore. 1995 Cross-clade neutralization of primary isolates of human immunodeficiency virus type 1 by human monoclonal antibodies and tetrameric CD4-IgG. J. Virol. 69:6609-6617.

29. Weiss, C. D., S. W. Barnett, N. Cacalano, N. Killeen, D. R. Littman, and J. M. White. 1996. Studies of HIV-1 envelope glycoprotein-mediated fusion using a simple fluorescence assay. AIDS 10:241-246.

30. Weissenhorn, W., A. Dessen, S. C. Harrison, J. J. Skehel, and D. C. Wiley. 1997. Atomic structure of the ectodomain from HIV-1 gp41. Nature 387:426-430.
31. Weissman, D., R. L. Rabin, J. Arthos, A. Rubbert, M. Dybul, R. Swofford, S. Venkatesan, J. M. Farber, and A. S. Fauci. 1997. Macrophage-tropic HIV and SIV envelope proteins induce a signal through the CCR5 chemokine receptor. Nature 389:981-985.

32. Wild, C., T. Oas, C. McDanal, D. Bolognesi, and T. Matthews. 1992. A synthetic peptide inhibitor of human immunodeficiency virus replication: correlation between solution structure and viral inhibition. Proc. Natl. Acad. Sci. USA 89:10537-10541.

33. Wu, L., N. P. Gerard, R. Wyatt, H. Choe, C. Parolin, N. Ruffing, A. Borsetti, A. A. Cardoso, E. Desjardin, W. Newman, C. Gerard, and J. Sodroski. 1996. CD4-induced interaction of primary HIV-1 gp120 glycoproteins with the chemokine receptor CCR-5. Nature 384:179-183.

34. Wyatt, R., E. Desjardin, U. Olshevsky, C. Nixon, J. Binley, V. Olshevsky, and J. Sodroski. 1997. Analysis of the interaction of the human immunodeficiency virus type 1 gp120 envelope glycoprotein with the gp41 transmembrane glycoprotein. J. Virol. 71:9722-9731.

35. Wyatt, R., J. Moore, M. Accola, E. Desjardin, J. Robinson, and J. Sodroski. 1995. Involvement of the V1/V2 variable loop structure in the exposure of human immunodeficiency virus type 1 gp120 epitopes induced by receptor binding. J. Virol. 69:5723-5733. 